\title{
Internal quantum efficiency and tunable colour temperature in monolithic white InGaN/GaN LED
}

\author{
Ilya E. Titkov*a, Amit Yadav ${ }^{\mathrm{a}}$, Vera L. Zerova ${ }^{\mathrm{a}}$, Modestas Zulonas ${ }^{\mathrm{a}}$, Andrey F. Tsatsulnikov ${ }^{\mathrm{b}}$, \\ Wsevolod V. Lundin ${ }^{\mathrm{b}}$, Alexey V. Sakharov ${ }^{\mathrm{b}}$, and Edik U. Rafailov ${ }^{\mathrm{a}, \mathrm{c}}$ \\ ${ }^{a}$ Photonics and Nanoscience Group, School of Engineering, Physics and Mathematics, University of \\ Dundee, Dundee, DD1 4HN, UK; \\ ${ }^{\mathrm{b}}$ Ioffe Physico-Technical Institute, Russian Academy of Sciences, Politekhnicheskaya str. 26, \\ 194021 St. Petersburg, Russia; \\ 'Photonics and Nanoscience Group, Aston Institute of Photonic Technologies, Aston University, \\ Birmingham, B4 7ET, UK
}

\begin{abstract}
Internal Quantum Efficiency (IQE) of two-colour monolithic white light emitting diode (LED) was measured by temperature dependant electro-luminescence (TDEL) and analysed with modified rate equation based on ABC model. External, internal and injection efficiencies of blue and green quantum wells were analysed separately. Monolithic white LED contained one green InGaN QW and two blue QWs being separated by GaN barrier. This paper reports also the tunable behaviour of correlated colour temperature (CCT) in pulsed operation mode and effect of self-heating on device performance.
\end{abstract}

Keywords: internal quantum efficiency, monolithic white LED, tunable CCT

\section{INTRODUCTION}

White LEDs tend to take their place in general lighting and are investigated intensively. ${ }^{1}$ Nowadays most of them are made using a phosphor coating that converts emissions from InGaN/GaN blue LED to yellow. The best reported devices have maximum external quantum efficiency (EQE) of $85 \%$ at the emission wavelength of $440 \mathrm{~nm}$ and luminous efficacy of $250 \mathrm{~lm} / \mathrm{W}$ obtained by the blue light conversion with a mixture of appropriate phosphors. ${ }^{2}$ Usual commercially available blue LEDs have EQE of about $40 \%$ at operation current $(350 \mathrm{~mA})$ and luminous efficacy of nearly $160 \mathrm{~lm} / \mathrm{W}$. This hybrid approach brings in disadvantages of unavoidable Stokes loss ${ }^{3,4}$ (about 20\%) occurring in phosphor and an addition of fabrication step in the device process.

To avoid this drawback various approaches of hybrid and monolithic white LEDs are investigated: different shape and size nanostructures ${ }^{5,6}$, QW light converter ${ }^{7}$, dichroic filter ${ }^{8}$, DBR resonant-cavity ${ }^{9}$ etc. A monolithic approach of few quantum wells emitting at different wavelengths can be also used to generate white light. ${ }^{10,11}$ From the first noted monolithic white LEDs ${ }^{10}$ they are being developed for over 10 years. ${ }^{10-16}$ Nevertheless, the EQE, colour rendering index (CRI) and CCT at sufficient luminous flux still need improvements. ${ }^{12,16}$ The main problem is that the IQE is low for yellow-green emitting InGaN QWs. ${ }^{10}$ Another crucial problem is the non-uniform carrier injection among the different InGaN QWs constituting the LED active region. Due to this the chromaticity coordinates of monolithic white LEDs strongly depend on the injection current. ${ }^{13}$ To see the way through and meet the goals of efficiency and colour parameters there is a need of innovation and optimization at each level of device technology from material growth to packaging.

New approach to monolithic white LED was recently suggested ${ }^{14,15,16}$. The authors first used short-period superlattice (SPSL) to improve free carriers injection between blue and green QWs, but maximal EQE was less than 7\%. The paper ${ }^{14}$ claimed there are two reasons for the poor efficiency of the monolithic white LEDs: the low internal quantum efficiency and non-uniform carrier injection into various QWs. Nevertheless, direct investigation of IQE or injection efficiencies (IE) in such structures has never been reported before. This paper is targeted to complete this study with more details and at extended operation currents and also reports the tunable behaviour of CCT in pulse operation mode at extremely high currents up to $2 \mathrm{~A}$.

Gallium Nitride Materials and Devices IX, edited by Jen-Inn Chyi, Yasushi Nanishi, Hadis Morkoç, Joachim Piprek, Euijoon Yoon, Hiroshi Fujioka, Proc. of SPIE Vol. 8986, 89862A · @ 2014 SPIE CCC code: $0277-786 \mathrm{X} / 14 / \$ 18 \cdot$ doi: $10.1117 / 12.2040086$ 


\section{SAMPLE STRUCTURE}

The white LED structure under study was similar to the structure used before ${ }^{14}$ just without SPSL in the barrier between blue and green QWs (Figure 1).

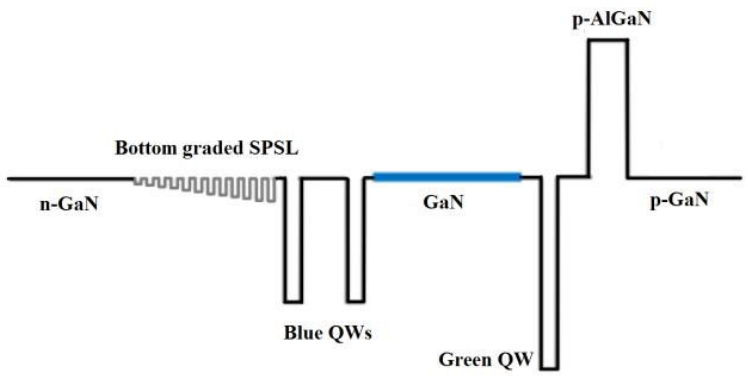

Figure 1. Conduction band diagram of the White LED structure.

The LED sample was grown by MOCVD on c-plane sapphire substrate with an underlying GaN buffer layer which supports the $\mathrm{n}-\mathrm{GaN}$ layer of $10^{18} \mathrm{~cm}^{-3} \mathrm{Si}$-doping concentration. On top of this $\mathrm{n}-\mathrm{GaN}$ layer a bottom graded SPSL was grown. The graded SPSL thickness was $24 \mathrm{~nm}$ with an alternate $1 \mathrm{~nm}$ deposition $\mathrm{InGaN}$ and GaN making a 12 period SPSL of InGaN/GaN. These SPSLs were grown by conversion method described by W.V. Lundin et. al ${ }^{17}$. On top of the SPSL the structure supports a $12 \mathrm{~nm}$ i-GaN barrier layer before the deposition of QWs.

The bottom graded SPSL with i-GaN barrier layer supports two InGaN QWs with band gap energy corresponding to blue wavelength of the visible spectrum and a single InGaN QW with energy gap corresponding to green wavelengths of the spectrum. All the three QWs are spatially $8 \mathrm{~nm}$ apart from each other with i-GaN used to have that separation in space. The top green QW in two colour LED is followed by $8 \mathrm{~nm}$ i-GaN barrier layer, $18 \mathrm{~nm}$ AlGaN EBL layer and $180 \mathrm{~nm}$ pGaN layer.

The structure was processed as TM-27 $\left(680 \times 560 \mu \mathrm{m}^{2}\right)$ and MK-24 $\left(1310 \times 1310 \mu \mathrm{m}^{2}\right)$ chips developed by ScientificTechnological Centre of Microelectronics and Submicrometer Heterostructures, Russian Academy of Science. The chip design and its optical properties were carefully described previously. ${ }^{18}$

\section{EXPERIMENT}

A Labsphere "CDS-600" spectrometer accompanied with "LightMtrx" software and helium closed-cycle cryostat "Janis CCS-450" were used for this purpose. In order to determine EQE, we measured the electroluminescence (EL) intensity at various temperatures from 100 to $300 \mathrm{~K}$ in a wide range of operating currents, from $10^{-4}$ to $1 \mathrm{~A}$. To cover a large range of the measured radiant fluxes, from $1 \mathrm{nW}$ to $0.1 \mathrm{~W}$, we used photodetector exposure time from $1 \mathrm{~ms}$ to $5 \mathrm{~s}$ and ND1-4 filters for light intensity attenuation. EQE was measured in integrating sphere and cryostat at room temperature (RT); after that the optical alignment was not changed at other temperatures. In order to obtain absolute values of EQE for temperatures other than RT, we used the normalization factors calculated for the RT data. The absence of any media adjacent to the chip surface is essential for correct temperature- and intensity-dependent electroluminescence (T-IDEL) measurements. Blue and green bands optical powers were measured separately by selecting the corresponding digital aperture. Internal and injection efficiencies (IQE and IE) of each band were evaluated by fitting procedure with $\mathrm{ABC}$ model and following the method reported by Rue et.al. ${ }^{19}$

To examine CCT tunability of the white LED we measured emission spectra in the continuous wave (CW) and pulse modes using current pulses from "Agilent 8114A" pulse generator. We varied the pulse widths from $100 \mathrm{~ns}$ to $100 \mu \mathrm{s}$ and duty cycle from $1 \%$ to $95 \%$. The current-voltage (I-V) characteristics of the LEDs were measured by the "Keithley 4200 " semiconductor characterisation system at the room temperature. 


\section{RESULTS AND DISCUSSION}

\subsection{Current-Voltage characteristic}

Quality of the LED chip can be estimated from its I-V characteristic. Figure 2 shows the I-V characteristic measured with MK-24 white LED chip at room temperature.

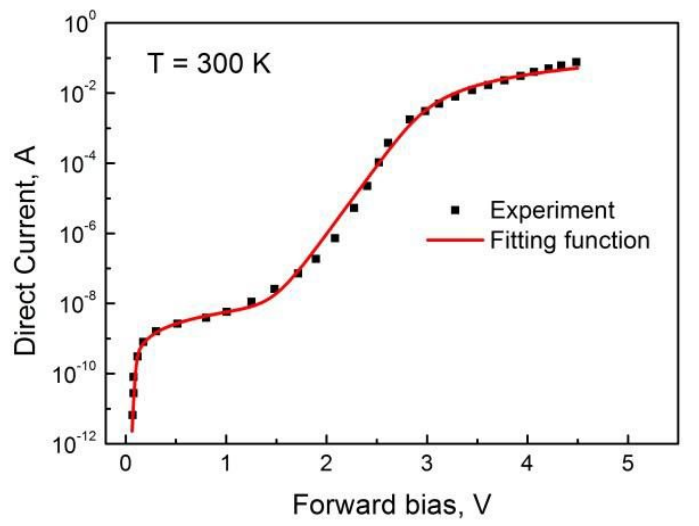

Figure 2. Current-Voltage characteristic of the white LED (symbols) and fitting function (solid line).

For the leakage level analysis we used a fitting procedure with one parallel and one series resistance equivalent scheme. The parameters obtained from the fitting are the following: parallel resistance $R_{p}=170 \mathrm{MOhm}$; series resistance $\mathrm{R}_{\mathrm{s}}=25 \mathrm{Ohm}$; ideality factor $\mathrm{f}=4.3$; saturation current $\mathrm{I}_{0}=1.5^{*} 10^{-14} \mathrm{~A}$. The quite high parallel resistance value points to the low leakage level that is negligible for IQE and IE analysis. The series resistance shows normal free carrier concentration in the p-, n- claddings. Such a high ideality factor can be attributed to the undoped GaN barriers inside the active region. ${ }^{20,21}$

\subsection{Efficiency analysis}

The method of IQE evaluation from $\mathrm{EQE}$ based on the $\mathrm{ABC}$-model requires a balance between generation of charge carriers by injection and their recombination. A possible misbalance is normally attributed to IE $<1$ in the case of negligible parallel leakage. This is absolutely correct for a single QW. Multiple quantum wells may be pumped nonuniformly, and for analysis we have to know how exactly carriers passing through the contacts redistribute between different quantum wells. For blue and green QWs of the structure under study nonuniformity was considered with partial contributions to the EL emission. For both blue QWs we assumed injection uniformity.

In the previous work ${ }^{18}$ authors studied light propagation and extraction from the LED die by 3D ray-tracing accounting for the optical properties of all elements of the chip design. In particular, the reflectivity of the $\mathrm{n}$ - and p-electrodes was assumed to be of $87 \%$ at normal incidence. Considering that Ag-based bottom electrode uniform reflectivity and matted sapphire surface mostly contribute to the whole light extraction efficiency (LEE) we can assume the same LEE for the blue and green QWs. 

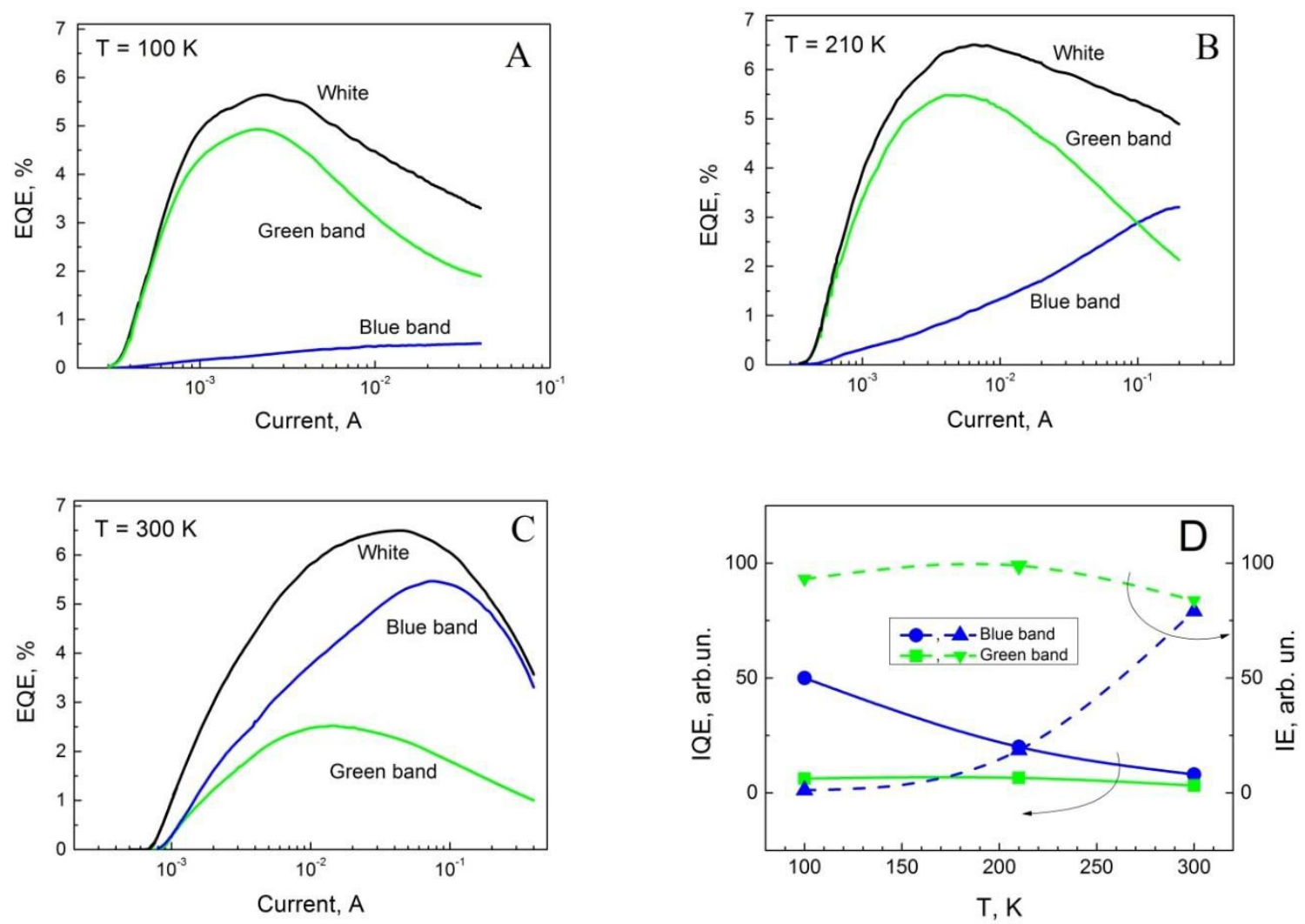

Figure 3. EQE of the monolithic white LED (TM) vs. currents at different temperatures: A - 100K; B - 210K and C - 300K; D presents evaluated Internal and Injection Efficiencies (IQE and IE) vs. temperature.

Figure 3 (A, B, C) shows measured EQE of the monolithic white LED vs. current at different temperatures. The white $\mathrm{EQE}$ is resulted from the balance of the green and blue bands efficiencies, which were measured separately. IQE and IE were evaluated by fitting of EQE(I) experimental curve with the method described before. ${ }^{19,22}$

Figure 3 (D) presents the evaluated IQE and IE vs. temperature for the green and blue bands. From these curves one can see that IQE and IE of the blue band strongly depend on the temperature, whereas for the green band they are almost constant. Such behaviour can be connected with different depths of the green and blue wells, as well as with impeded hole transport to the blue QWs. One more thing is coming from these graphs is that for the blue band the temperature affects the IE stronger than IQE.

\subsection{CCT and spectral data analysis}

Here we obtained CCT and green/blue peaks ratio of the white LEDs at extended operation currents from $100 \mathrm{~mA}$ to $2 \mathrm{~A}$ providing the luminous flux up to $2.3 \mathrm{Lm}$. To examine CCT tunability of the white LED we measured emission spectra in $\mathrm{CW}$ and pulse modes using current pulses from $100 \mathrm{~ns}$ to $100 \mu$ s and varying duty cycle from $1 \%$ to $95 \%$. All the pulse measurements were taken into integration sphere at the room temperature.

\subsubsection{Overheated mode}

We considered the Duty cycle $>1 \%$ as an overheated mode. Figure 4 demonstrates strong dependence of CCT on the duty cycle value. The CCT changes more than three times vs. Duty cycle at $300 \mathrm{~mA}$ pulse operation current. From figure 4 one can see that dependence of the CCT on the pulse width is negligible. 


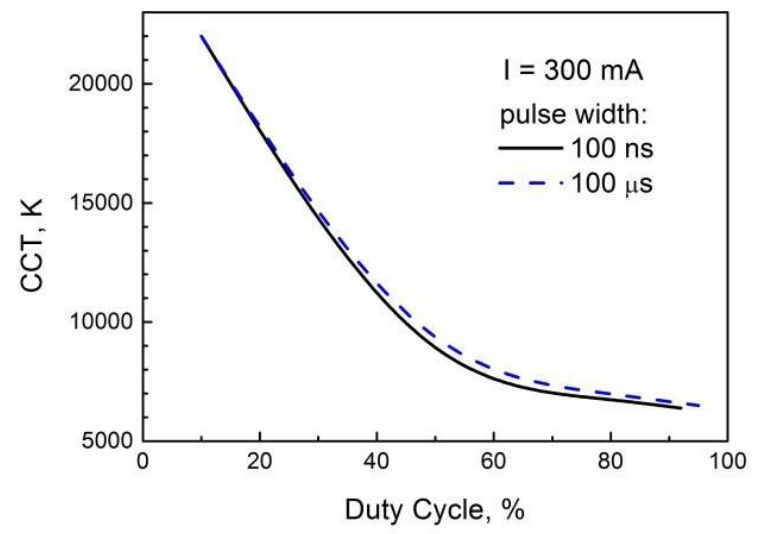

Figure 4. CCT vs. Duty Cycle of the white LED measured in the integration sphere at $100 \mathrm{~ns}$ and $100 \mu$ s pulse durations.
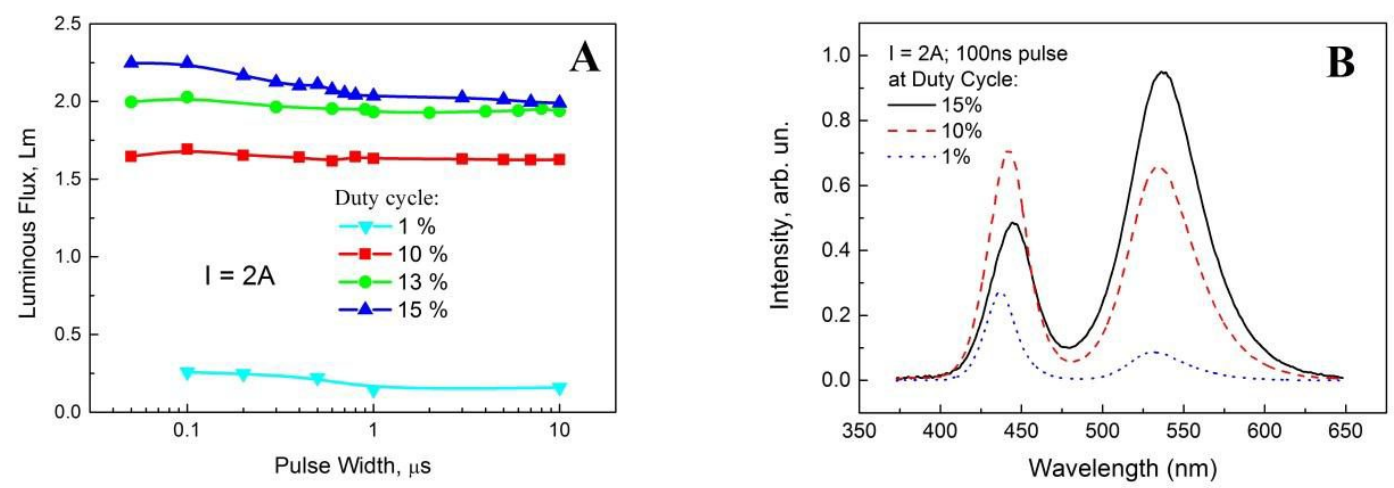

Figure 5. A - Luminous flux vs. pulse width measured at different Duty cycles and 2A driving current. B - EL emission spectra at $2 \mathrm{~A}, 100 \mathrm{~ns}$ pulse current and various duty cycles.

Figures $5(\mathbf{A}, \mathbf{B})$ demonstrates a possibility to keep the luminous flux independent from pulse width in the range of more than two orders.
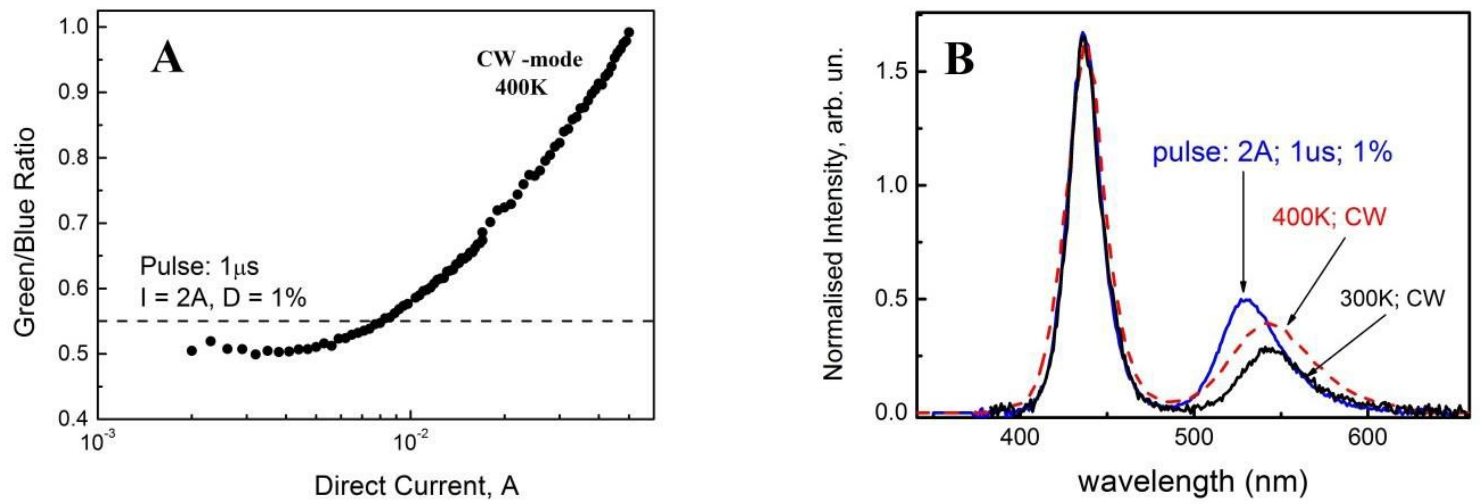

Figure 6. A - Experimental data of colour mixing (green/blue bands ratio) vs. direct current (solid circles) measured at $400 \mathrm{~K}$. The dash line shows the level of colour mixing with pulse mode at the room temperature; $\mathrm{I}=2 \mathrm{~A}, 1 \mu$ s pulse width and $1 \%$ Duty cycle. B - Normalised EL spectra at the cross point between the symbol and dash lines on the Figure 6 (A). CW EL was measured at $8 \mathrm{~mA}$ direct current. 
We also observed that green/blue peaks ratio has the same values for $\mathrm{CW} 8 \mathrm{~mA}$ at $\mathrm{T}=127^{\circ} \mathrm{C}(400 \mathrm{~K})$ and for pulse mode $1 \%$ Duty Cycle, I = $2 \mathrm{~A} ; 200 \mathrm{~ns}-1 \mu$ s pulse width, Figure 6 (A). If suppose the spectra transformation is caused by overheating, that means at maxim pulse current $(2 \mathrm{~A})$ the active region works nearly at temperature of about $127^{\circ} \mathrm{C}$. On the other hand figure 6 (B) shows the green peak is narrower and shifted left from the $400 \mathrm{~K}$ peak. This is an indirect evidence of the lower temperature of active region at the present pulse regime $\left(<127^{\circ} \mathrm{C}\right)$ and supports the idea of carrier dynamics influence on the colour mixing.

\subsubsection{Non-overheated mode}

To provide non-overheated regime we investigated the colour mixing behaviour at $1 \%$ duty cycle. From figure 7 one can see, that even at $1 \%$ duty cycle the colour mixing, and hence CCT, can be tunable in about three times by pulse width and current amplitude modulation. For example, increase of current in the range of 0.1-2A with simultaneous decreasing pulse width to keep average electric power constant, allows changing of the green/blue ratio in the wide range from $\sim 0.8$ to $\sim 0.2$. We suppose it should be possible also keeping input power and luminous flux as a constant.

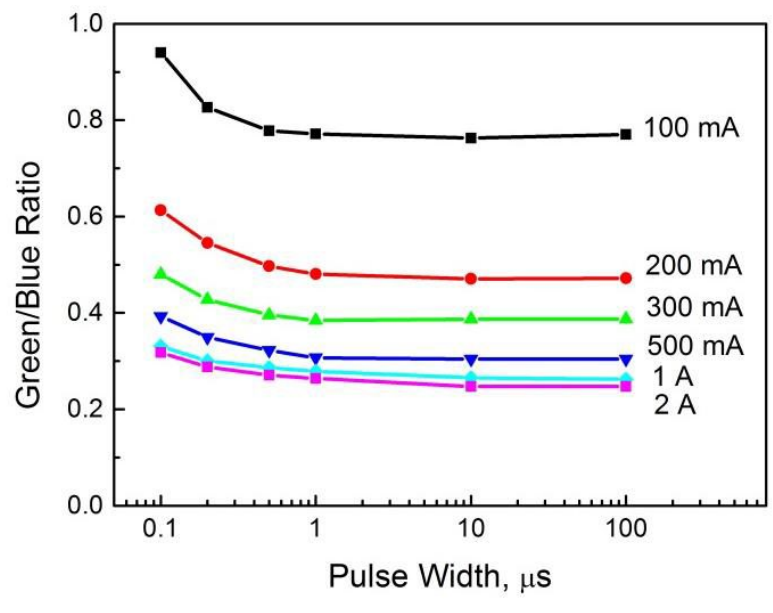

Figure 7. Green/Blue bands ratio vs. pulse width measured at different currents and $1 \%$ Duty cycle. The figure demonstrates colour mixing tunability even at non-overheated mode.

\section{CONCLUSION}

EQE of two-colour monolithic white LEDs were measured by temperature dependant electro-luminescence. IQE and IE were estimated from the fitting by $\mathrm{ABC}$ model. Whereas maximal IQE of the green $\mathrm{QW}$ in the white structure is really lower than the blue one, injection efficiencies are almost the same at the room temperature. Summarising these results we can conclude that $\mathrm{ABC}$ model is applicable for two-colour structures and can be useful for rough estimations and watching for IQEs and IEs balance with temperature.

The green/blue ratio, and hence CCT, can be tunable in about three times by pulse width and current amplitude modulation. We suppose it should be possible also keeping input power and luminous flux as a constant. This is a subject of the future investigations. Finally we have got new knowledge about two-colour structures at extremely high operation currents. We believe it could be useful for the future development of monolithic white sources.

\section{AKNOWLEDGEMENTS}

This work was supported by European Union FP7, NEWLED project, Grant number 318388. Authors wish to thank Sergey Yu. Karpov (STR Group Ltd.) for very productive discussion. 


\section{REFERENCES}

[1] Crawford, M. H., "LEDs for solid-state lighting: performance challenges and recent advances," IEEE J. Sel. Top. Quantum Electron. 15, 1028-1040 (2009).

[2] Narukawa, Y., Ichikawa, M., Sanga, D., Sano, M., and Mukai, T., "White light emitting diodes with super high luminous efficacy,” J. Phys. D: Appl. Phys. 43, 354002 (2010).

[3] Schubert, E. F., and Kim, J. K., Science, "Solid-state light source getting smart," Science 308 (5726), 12741278 (2005).

[4] Schubert, E. F., [Light-Emitting Diodes], Cambridge University Press, Cambridge, England, (2003).

[5] Kim, T., "A nanopyramid structure for monolithic white-light LEDs," SPIE Newsroom, Jan. 30 (2013).

[6] Ko, Y.-H., Kim, J.-H., Jin, L.-H., Ko, S.-M., Kwon, B.-J., Kim, J., Kim, T., Cho, Y.-H., "Electrically driven quantum dot/wire/well hybrid light-emitting diodes," Adv. Mater. 23(45), 5364-5369 (2011).

[7] Damilano, B., Trad, N., Brault, J., Demolon, P., Natali, F. and Massies, J., "Colour control in monolithic white light emitting diodes using a (Ga,In)N/GaN multiple quantum well light converter," Phys. Status Solidi A 209(3), 465-468(2012).

[8] Oh, J. H., Oh J. R., Park, H. K., Sung, Y.-G., and Do, Y. R., "Highly-efficient, tunable green, phosphorconverted LEDs using a long-pass dichroic filter and a series of orthosilicate phosphors for tri-color white LEDs," Optics Express 20(S1), A1-A12 (2012).

[9] Yu, C., Lirong, H., and Shanshan, Z., "Monolithic white LED based on AlxGa1-xN/InyGa1-yN DBR resonantcavity," Journal of Semiconductors 30, 014005 (2009).

[10] Yamada, M., Narukawa, Y., and Mukai, T., "Phosphor Free High-Luminous-Efficiency White Light-Emitting Diodes Composed of InGaN Multi-Quantum Well,” Jpn. J. Appl. Phys. 41, L246-L248 (2002).

[11] Damilano, B., Grandjean, N., Pernot, C. and Massies, J., "Monolithic white light emitting diodes based on InGaN/GaN multiple-quantum wells," Jpn. J. Appl. Phys. 40, L918-L920 (2001).

[12] Lu, C. F., Huang, C. F., Chen, Y. S., Shiao, W. Y., Chen, C. Y., Lu, Y. C., and Yang, C. C., "Phosphor-Free Monolithic White-Light LED," IEEE Journal of Selected Topics in Quantum Electronics 15, 1210-1217 (2009).

[13] Dalmasso, S., Damilano, B., Pernot, C., Dussaigne, A., Byrne, D., Grand- jean, N., Leroux, M., and Massies, J., "Injection Dependence of the Electroluminescence Spectra of Phosphor Free GaN-Based White Light Emitting Diodes," Phys. Status Solidi A 192, 139-143 (2002).

[14] Tsatsulnikov, A. F., Lundin, W. V., Sakharov, A. V., Zavarin, E. E., Usov, S. O., Nikolaev, A. E., Kryzhanovskaya, N. V., Synitsin, M. A., Sizov, V. S., Zakgeim, A. L., and Mizerov, M. N., "A monolithic white LED with an active region based on InGaN QWs separated by short-period InGaN/GaN superlattices," Semiconductors 44(6), 808-811 (2010).

[15] Tsatsulnikov, A. F., Lundin, W. V., Sakharov, A. V., Zavarin, E. E., Usov, S. O., Nikolaev, A. E., Kryzhanovskaya, N. V., Sizov, V. S., Synitsin, M. A., Yakovlev, E. V., Chernyakov, A. E., Zakgeim, A. L., Cherkashin, N. A. and Hytch, M., "InGaN/GaN short-period superlattices: synthesis, properties, applications," Phys. Status Solidi C 8, 2308-2310 (2011).

[16] Ustinov, V. M., Tsatsulnikov, A. F., Lundin, V. V., Sakharov, A. V., Nikolaev, A. E., Zavarin, E. E., Zakgeim, A. L., Chernyakov, A. E., Mizerov, M. N., Cherkashin, N. A., and Hytch, M., "Monolithic White LEDs: Approaches, Technology, Design," Journal of Surface Investigation. Xray, Synchrotron and Neutron Techniques 6, 501-504 (2012)

[17] Lundin, W.V., Nikolaev, A.E., Sakharov, A.V., Zavarin, E.E., Valkovskiy, G.A., Yagovkina, M.A., Usov, S.O., Kryzhanovskaya, N.V., Sizov, V.S., Brunkov, P.N., Zakgeim, A.L., Cherniakov, A.E., Cherkashin, N.A., Hytch, M.J., Yakovlev, E.V., Bazarevskiy, D.S., Rozhavskaya, M.M., and Tsatsulnikov, A.F., "Single quantum well deep-green LEDs with buried InGaN/GaN short-period superlattice," Journal of Crystal Growth 315, 267 (2011).

[18] Chernyakov, A. E., Bulashevich, K. A., Karpov, S. Yu., and Zakgeim, A. L., "Experimental and theoretical study of electrical, thermal, and optical characteristics of InGaN/GaN high-power flip-chip LEDs," Phys. Status Solidi A 210, 466-469 (2013).

[19] Rue, H. Y., Kim, H. S., and Shim, J. I., "Rate equation analysis of efficiency droop in InGaN light-emitting diodes," Appl. Phys. Lett. 95, 081114 (2009).

[20]Zhu, D., Xu, J., Noemaun, A. N., Kim, J. K., Schubert, E. F., et al., "The origin of the high diode-ideality factors in GaInN/GaN multiple quantum well light-emitting diodes,” Appl. Phys. Lett. 94, 081113 (2009). 
[21] Shah, J. M., Li, Y.-L., Gessmann, Th., and Schubert, E. F., "Experimental analysis and theoretical model for anomalously high ideality factors ( $\mathrm{n}>>$ 2.0) in AlGaN/GaN p-n junction diodes," J. Appl. Phys. 94, 2627 (2003).

[22] Titkov, I. E., Sannikov, D. A., Park, Y.-M., and Son, J.-K., "Blue light emitting diode internal and injection efficiency,” AIP Advances 2, 032117 (2012). 